\title{
MOBILIDADE DO ZIRCÔNIO NA BAUXITIZAÇÃO DE ROCHAS ALCALINAS DO MACIÇO DE POÇOS DE CALDAS, MINAS GERAIS
}

\author{
FRANCOIS SOUBIÈS*, ADOLPHO J. MELFI**, JEAN DELVIGNE*** $\mathrm{e}$ \\ IVONETE A. SARDELA*****
}

\begin{abstract}
ZIRCONIUM MOBILITY DURING THE LATERITIC WEATHERING OF ALKALINE ROCKS OF THE POÇOS DE CALDAS COMPLEX MINAS GERAIS, BRAZIL. Some bauxitic profiles, developed from alkaline rocks of the Poços de Caldas, Minas Gerais, Brazil complex were studied. The preliminary results show that nearly $40 \%$ of the total zirconium existing in the rock was leached out of the profile during the bauxitization process. On the other hand, it became evident that only a small part of the bauxitic residual zirconium is tied to zircon. Most of it is associated with the bauxitic ferruginous phase as colloidal hydroxides mixtures.
\end{abstract}

Keywords: Zirconium, bauxite, laterization, weathering.

RESUMO Estudos mineralógicos e geoquímicos efetuados nos perfis de alteração do depósito de bauxita do Morro do Cristo (Poços de Caldas, Minas Gerais) evidenciam que cerca de $40 \%$ do zircônio contido na rocha alcalina são lixiviados do perfil durante o processo de bauxitização. Por outro lado, os resultados obtidos mostram ainda que apenas uma pequena parte do zircônio residual da bauxita encontra-se sob a forma de zircão. A quase totalidade deste elemento associa-se à fase ferruginosa goethítica da bauxita sob a forma de geles ferro-alumínio-titânio-zirconíferos.

Palavras-chaves: Zircônio, bauxita, laterização, intemperismo.

INTRODUÇÃO Apesar do comportamento geoquímico do $\mathrm{Zr}$ no ciclo supérgeno não estar ainda perfeitamente estabelecido (Milnes \& Fitzpatrick 1989) e da existência de evidências de sua mobilização no curso do intemperismo, sob forma de complexos orgânicos (Ronov et al 1961, Kimura \& Swindale 1967), é comum considerá-lo como um elemento de grande estabilidade e praticamente imóvel no manto superficial de alteração. Dessa forma, o $\mathrm{Zr}$ acumula-se residualmente nos perfis de intemperismo (Goldschmidt 1937,1954, Degenhardt 1957, Adams \& Richardson 1960, Zeissink 1971, Levinson 1980).

Na realidade, esta aparente imobilidade pode ser explicada, de um lado, pela fraca mobilidade do $\mathrm{Zr}^{4+}$, dentro de uma larga variação de $\mathrm{pH}$ e Eh (Levinson 1980, Brookins 1988), e, por outro lado, pelo fato do $\mathrm{Zr}$, geralmente, ocorrer nas rochas concentrado em um único mineral, o zircão, que quando não está metamítico apresenta alta resistência à dissolução (Goldich 1938, Milnes \& Fitzpatrick 1989).

Durante muito tempo, os altos teores de $\mathrm{Zr}$ encontrados em bauxitas eram explicados única e exclusivamente pela presença de zircão nesses produtos lateríticos (Frederickson 1948, Zakrutkin \& Shvetsiva 1974 etc.).

Por outro lado, sabe-se que, em muitos casos, somente o zircão, não poderia explicar os altos teores de $\mathrm{Zr}$ encontrados em bauxitas. Os trabalhos de Degenhardt (1969) e Beneslavskii (1963) mostram que, nas bauxitas, o Zr, além de se concentrar no zircão, ocorre, também, provavelmente, como óxidos e hidróxidos coloidais, ou adsorvido na forma iônica em minerais secundários ou, ainda, substituindo isomorficamente o ferro e o alumínio em óxidos ou hidróxidos. Zakrutkin \& Shvetsiva (1974), estudando bauxitas das montanhas do sul do Timan (URSS), sugerem que o $\mathrm{Zr}$ pode estar presente sob três formas diferentes: no zircão, em óxidos hidratados de zircônio e, predominantemente, em substituições isomórficas na boehmita e na caolinita.
Desta forma, vê-se que, além do zircão residual, poderiam ocorrer nos mantos de intemperismo, especialmente nas bauxitas, fases zirconíferas secundárias, ainda mal identificadas, oriundas de diferentes portadores primários, cuja neoformação indicaria certa mobilização do $\mathrm{Zr}$ nos meios superficiais.

Em Poços de Caldas, depósitos de bauxita ricos em $\mathrm{Zr}$ são desenvolvidos sobre rochas alcalinas com altos teores neste elemento (cerca de $1.500 \mathrm{ppm}$ ) e quase isentas de zircão. Com o objetivo de caracterizar o comportamento geoquímico do Zr durante o processo de bauxitização e sua eventual localização na bauxita, estudos mineralógicos e químicos foram realizados em perfis do depósito do Morro do Cristo, desenvolvidos a partir de tinguaítos (Fig. 1).
A BAUXITA DO DEPÓSITO DO MORRO DO CRISTO Contexto geológico e petrográfíco $O$ maciço alca- líno de Poços de Caldas, de idade cretácica superior (Amaral et al 1967), forma um grande corpo circular, com aproxima- damente $800 \mathrm{~km}^{2}$ de área, intrusivo em rochas pré-cambrianas. Este maciço destaca-se nitidamente na paisagem montanhosa do embasamento pré-cambriano, por marcado desnível topo- gráfico de cerca de $300 \mathrm{~m}$.
Do ponto de vista petrográfico, o maciço é formado do- minantemente por tinguaítos, fonolitos e nefelina sienitos, associados a lavas ankaramíticas, brechas, e rochas piroclás- ticas (Ellert 1959). Os tinguaítos e os fonolitos são os termos litológicos mais importantes na gênese dos depósitos de bau- xita, não somente pelo fato de suas composições químicas e mineralógicas favorecerem a bauxitização, mas, principal- mente, pela sua grande expressão areolar e estrutura.
O depósito do Morro do Cristo ocorre na borda do maciço de Poços de Caldas, onde predomina uma topografia altamente acidentada, com grandes desníveis, altimétricos e vertentes abruptas. As condições de drenagem são excelentes e possibi- litam forte e rápida alteração da rocha alcalina. Como resulta-

\footnotetext{
* ORSTOM, UR/IG/Laboratoire de Minéralogie, Université Paul Sabatier, 39, Alices Jules Guesde, 31000 Toulouse, Franca

** Departamento de Geofísica, Instituto Astronômico e Geofísico, Universidade de São Paulo, Caixa Postal 30627, CEP 01051, São Paulo, SP, Brasil

*** ORSTOM, UR/IG/Laboratoire de Pétrologie de la Surface et de Geologic Dynamique, Université d'Aix-Marseille III, Av. Escadrille Normandie-Niemen, 13397 Marseille, França 


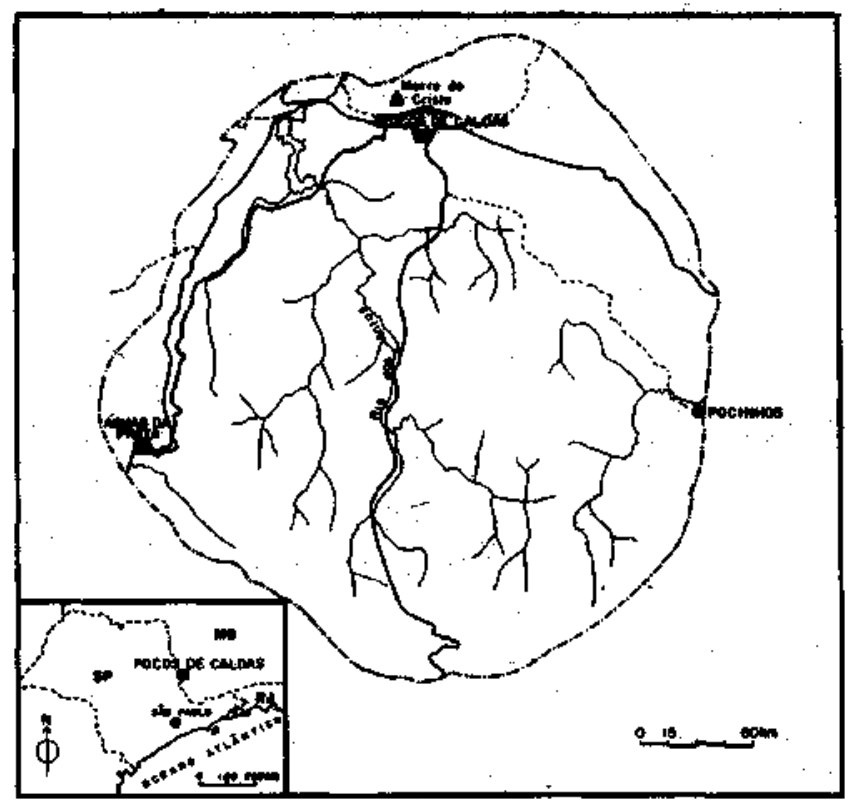

Figura 1 - Mapa de bocalizacão da area estudada Figure 1 - Location map of the studied area

do, tem-se a formação de perfil bauxítico em contato direto com a rocha original; rico em $\mathrm{Al}$ e pobre em $\mathrm{Si}$.

A rocha mãe do depósito do Morro do Cristo é um tinguaíto, caracterizado por apresentar textura afanítica ou fanerítica muito fina, cor cinza-esverdeado escuro e com poucos fenocristais visíveis a olho nu. É comum a presença de numerosas fraturas, algumas preenchidas por material totalmente argilifícado.

Microscopicamente, esta rocha é constituída por um mosaico de grãos finos (algumas dezenas de $\mu \mathrm{m}$ ) de feldspato e nefelina, aproximadamente equidimensionais, que, por vezes, se apresentam idiomorfos e, neste caso, são de maiores dimensões (até 0,5 mm). Inúmeros pequenos prismas de egirina-augita (200 x $100 \mu \mathrm{m}$ em média) estão dispersos nesse fundo matricial e constituem mais ou menos $20 \%$ do volume total da rocha. Igualmente dispersos na matriz são encontrados cristais maiores $(0,5$ a $1,0 \mathrm{~mm})$ de "giannettita" e de um anfibólio sódico (ferro-edentta), que constituem cerca de $5 \%$ do volume total da rocha. Estes cristais são sempre poiquilíticos e englobam numerosos pequenos cristais de feldspato e nefelina da matriz. Zircão existe, mas seus cristais são extremamente raros, ocorrendo como traços.

Quimicamente, estes tinguaítos apresentam um caráter mais agpaítíco que miasquítico, o que é reforçado pelos altos teores em $\mathrm{Zr}$, Nb e TR (Tab. 1).

$\mathrm{O}$ alto teor em $\mathrm{Zr}$ encontrado no tinguaíto não pode ser explicado pela presença de zircão, visto que, nas cinco amostras estudadas, apenas quatro diminutos cristais deste mineral foram encontrados. O estudo por microssonda eletrônica (CAMECA SX 50, equipada com espectrômetrps WDS) mostrou que, de todos os minerais formadores do tinguaíto, apenas o anfibólio sódico e, sobretudo, a "giannettita" possuem Zr em sua constituição.

O anfibólio sódico apresenta composição química que permite classificá-lo como ferro-edenita, com baixo teor em Zr (Tab. 2).

O principal portador de $\mathrm{Zr}$ no tinguaíto do Morro do Cristo apresenta todas as características ópticas da "giannettita", mineral descrito em Poços de Caldas por Guimaiies (1948). Segundo este autor, a "giannettita" mostra uma certa semelhança com hiortdahlita, um flúoro-zirconossilicato cálcio-sódico do grupo da wôhlerita-lavenita (Strunz 1966). Entretanto, os insuficientes dados químicos obtidos por Guimarães (1948) e a ausência de dados de difração de raios X não permitiram,
Tabela1 - Composição química média (três amostras) do tinguaito do Morro do Cristo

Table 1 - Mean chemical composition (three samples) of the "Morro do Cristo" tinguaite

\begin{tabular}{|c|c|c|c|}
\hline Oxido: & \% peso & Oxidos & * paso \\
\hline $\mathrm{SIO}_{2}$ & 50,30 & $\mathrm{~K}_{2} \mathrm{O}$ & 7,40 \\
\hline $\mathrm{THO}_{2}$ & 0,58 & $\mathrm{P}_{2} \mathrm{O}_{6}$ & 0,06 \\
\hline $\mathrm{Al}_{2} \mathrm{O}_{3}$ & 19,10 & $\mathrm{ZrO}_{2}$ & 0,12 \\
\hline $\mathrm{F}_{\theta_{2}} \mathrm{O}_{3}$ & $\mathbf{3 , 6 1}$. & $\mathrm{V}_{2} \mathrm{O}_{5}$ & 0,02 \\
\hline $\mathrm{MnO}_{2}$ & 0,38 & $\mathrm{Nb}_{2} \mathrm{O}_{5}$ & 0,08 \\
\hline $\mathrm{MpO}$ & 0,30 & $\mathrm{CeO}_{2}$ & 0,05 \\
\hline $\mathrm{CaO}$ & 1,36 & $\mathrm{Lag}_{2} \mathrm{O}_{3}$ & 0,02 \\
\hline $\mathrm{Ne}_{2} \mathrm{O}$ & 5,31 & Totel & 96,68 \\
\hline
\end{tabular}

até hoje, a perfeita caracterização desse material (Embrey \& Fuller 1980). Além disso, nesse grupo estão reunidos dezenas de sorossillicatos de $\mathrm{Ca}, \mathrm{Na}, \mathrm{Ti}, \mathrm{Nb}, \mathrm{Zr}$, com composições químicas semelhantes, cuja fórmula geral, segundo Aarden \& Gittins (1974) e Merlino \& Perchiasi (1988), pode ser escrita: $\mathrm{X}_{16}\left(\mathrm{Si}_{2} \mathrm{O}_{7}\right) 4(\mathrm{O}, \mathrm{OH}, \mathrm{F})_{8}$.

Apesar da grande quantidade de inclusões de feldspatos e feldspatóides dificultar a caracterização química da "giannetita", algumas análises químicas parciais, obtidas por microssonda eletrônica durante este trabalho, são apresentadas na tabela 3. Dos elementos terras raras, apenas o Ce foi analisado, podendo estar presentes $\mathrm{La}, \mathrm{Nb}$ etc, o que aproximaria os totais da análise para $100 \%$.

Pode-se constatar quê as análises realizadas correspondem à fórmula cristaloquímica dos minerais do grupo da wöhlerita-lavenita. Por exemplo, considerando-se a análise $\mathrm{n}^{\mathbf{0}} 6$ (Tab. 3), um cálculo com base (8Si) leva a um total de cations, de $\mathrm{O}$ e de $\mathrm{F}$, compatível com a fórmula dos minerais desse grupo:

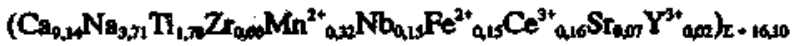

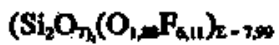

Este fato, juntamente com os dados ópticos obtidos por Guimarães (1948), sugere que a "giannettita" tem boa probabilidade de pertencer a esta família de minerais, mas não permite afirmar, sobretudo pela ausência de dados difratométricos de raios $\mathrm{X}$, que se trata de hiortdahlita, de um outro mineral do grupo, ou ainda de um novo mineral.

Bauxitização dos tinguaitos As condições litológicas, topográficas e de drenagem existentes no Morro do Cristo são favoráveis ao desenvolvimento de notáveis perfis bauxíticos, caracterizados por uma bauxitização direta, muito rápida e já totalmente desenvolvida a poucos milímetros da rocha completamente fresca (Fig. 2).

Trata-se de perfis altamente evoluídos, com altos teores em alumina, pequenas proporções de argilominerais e não muito espessos (nestas regiões a erosão é intensa).

A alteração do tinguaíto tem início pela formação de «uma crosta de alteração, amarelo-avermelhada, de alta porosidade e composta essencialmente de gibbsita. Quantidades reduzidas de goethita são encontradas associadas à gibbsita. Produtos amorfos, com composição ferro-aluminosa, são comuns.

Em direção à superfície, diversas reorganizações estruturais, ligadas a transportes ou eliminação de matéria, podem 
Tabela 2 - Composição química, em \% peso, do anfibólio sádico (ferro-edenita). Média de quatro pontos analisados por microssonda eletrônica. Amostra PCMC-2.15

Table 2 - Chemical composition, in weight percent, of the sodic atnphibole (ferro-edenite). Mean values of four electron microprobe point analyses. Sample PCMC-2.15

\begin{tabular}{|c|c|c|c|c|c|c|c|c|c|c|c|c|c|c|c|}
\hline \multicolumn{16}{|c|}{ PCAC - 2.15 } \\
\hline $\mathbf{s} \omega_{2}$ & $\mathrm{TH}_{2}$ & $\mathrm{Al}_{2} \mathrm{O}_{3}^{-}$ & $\mathrm{F}: 0$ & Mno & MgO & $\mathrm{CaO}$ & $\mathrm{Na}_{2} \mathrm{O}$ & $\mathrm{K}_{2} \mathrm{O}$ & $\mathrm{ZrO}_{2}$ & $\mathrm{Nb}_{2} \mathrm{O}_{5}$ & $\mathrm{C}_{2} \mathrm{O}_{3}$ & $\mathbf{Y}$ & $\mathbf{F}$ & cl & $\mathbf{z}$ \\
\hline 47,54 & 1,96 & 1,69 & 24,60 & 2,13 & 3,58 & 7,27 & 6,65 & 4.13 & 0,15 & 0,03 & 0,02 & 0,00 & $0, \infty$ & 0,02 & 07,42 \\
\hline
\end{tabular}

Tabela 3 - Composição química, em \% peso, da "giannettita" (fluoro-zirconossilicato). Análise de vários cristais da amostra PCMC-2.15 (microssonda eletrônica) Table 3 - Chemical composition, in weight percent, of the "giannettite" (zirconium bearing fluorosilicate). Analyses of several crystals of the sample PCMC-2.15 (electron microprobe analyses)

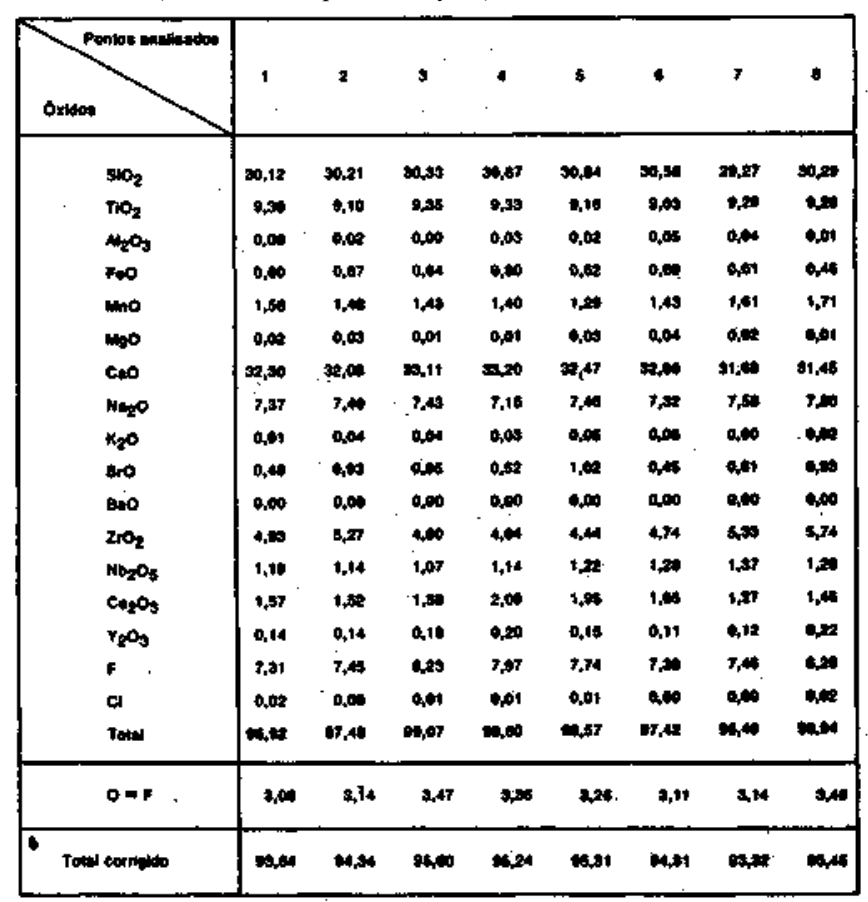

\section{Perifl Pontos de}

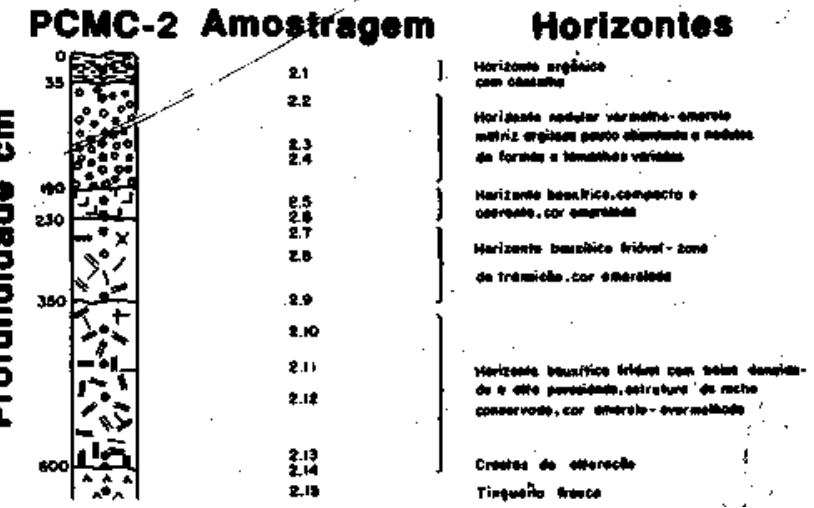

Figura 2 - Perfil do depósito bauxitico do Morro do Cristo Figure 2 - The Morro do Cristo bauxitic profile

ser observadas, porém sem que haja grandes mudanças na composição mineralógica e química global dos materiais.
Da base para o topo do perfil, foi posível distinguir três horizontes principais, com base em características morfológicas e físicas (Fig. 2).

Horizonte friável com estrutura preservada $\mathrm{Na}$ base do perfil, em contato abrupto com a rocha fresca, ocorre horizonte bauxitico amarelo-avermelhado, fortemente poroso, friável e com baixa densidade $\left(1,1\right.$ a $\left.1,4 \mathrm{~g} / \mathrm{cm}^{3}\right)$. Sua espessura é variável, apresentando em média $3 \mathrm{~m}$. Uma rede de fraturas planas (espessura milimétrica até centimétrica), herdada da rocha matriz, guarnecida por material mais avermelhado e com forte porosidade, divide este horizonte em blocos de dimensões decimétricas. Na parte inferior do horizonte, a estrutura da rocha-mãe encontra-se perfeitamente preservada nesses blocos. Na parte superior, começa, porém, a ocorrer certa redistribuição de matéria (mobilização do $\mathrm{Al}$ ), conduzindo a destruição parcial dessa estrutura e individuálização de meganódulos zonados (alteração esferoidal), com um córtex mais duro e compacto $\left(\mathrm{d}=1,5-1,7 \mathrm{~g} / \mathrm{cm}^{3}\right)$. A gibbsita, com goethita subordinada, é o componente essencial desse horizonte. Traços de caolinita, anatásio e halloysita podem ser encontrados. Zircão é esporádico, ocorrendo como traços em algumas amostras do horizonte. Geles amorfos são comuns.

Horizonte coerente denso Acima do horizonte friável, com contato transicional e espessura média de $2 \mathrm{~m}$, ocorre um horizonte mais coerente, denso $\left(\mathrm{d}=1,5-1,7 \mathrm{~g} / \mathrm{cm}^{3}\right)$ e compacto, com porosidade menos importante que no horizonte anterior e cor mais amarelada. Nesse nível, desaparecem as estruturas esferoidais geradas mais para a base do perfil, assim como qualquer vestígio da estrutura fina da rocha matriz. Argila de cor castanha, iluviada dos níveis superiores, preenche parte da porosidade desse material. A mineralogia é semelhante à do horizonte inferior, chegando a gibbsita a formar $90 \%$ do volume total do horizonte.

Horizonte nodular Mais próximo da superfície, abaixo de fino horizonte orgânico (cerca de $30 \mathrm{~cm}$ em média), vermelhoescuro, rico em cascalho, ocorre horizonte nodular, em geral pouco espesso (máximo de $1,5 \mathrm{~m}$ ), formado essencialmente de fragmentos do nível subjacente. Tais fragmentos, blocos ou nódulos, com tamanhos e formas muito variados, cor amarelada, rosada ou esbrahquiçada e densidade bastante alta (até $1,9 \mathrm{~g} / \mathrm{cm}^{3}$ ), são dispersos em matriz argilosa castanha e pouco abundante. Os nódulos apresentam composição mineralógica idêntica à dos horizontes inferiores e, portanto, rica em gibbsita, com goethita subordinada, enquanto a matriz argilosa é constituída dominantemente por caolinita e goethita.

Quimicamente (Tab. 4), o perfil bauxitico é caracterizado, em relação à rocha original, por notável diminuição dos teores de sílica ê dos elementos alcalinos e álcalinos terrosos, e enriquecimento de $\mathrm{Al}_{2} \mathrm{O}_{3}$ e $\mathrm{Fe}_{2} \mathrm{O}_{3}$, o que evidencia típica evolução ferralítica.

Nos dois horizontes inferiores (friável com estrutura preservada e coerente denso), a bauxita caracteriza-se, 
Tabela 4 - Análise química total do perfil bauxítico do Morro do Cristo. Determinações feitas nos laboratórios da Nuclebrás (Poços de Caldas) por espectrotnetria de plasma (ICP) a partir de amostras representativas das diferentes fácies encontradas no perfil

Table 4 - Chemical analyses of the Morro do Cristo bauxitic profile. Induced coupled plasma (ICP) analyses (Nuclebrás, Poços de Caldas) from the various weathering facies observed in the profile

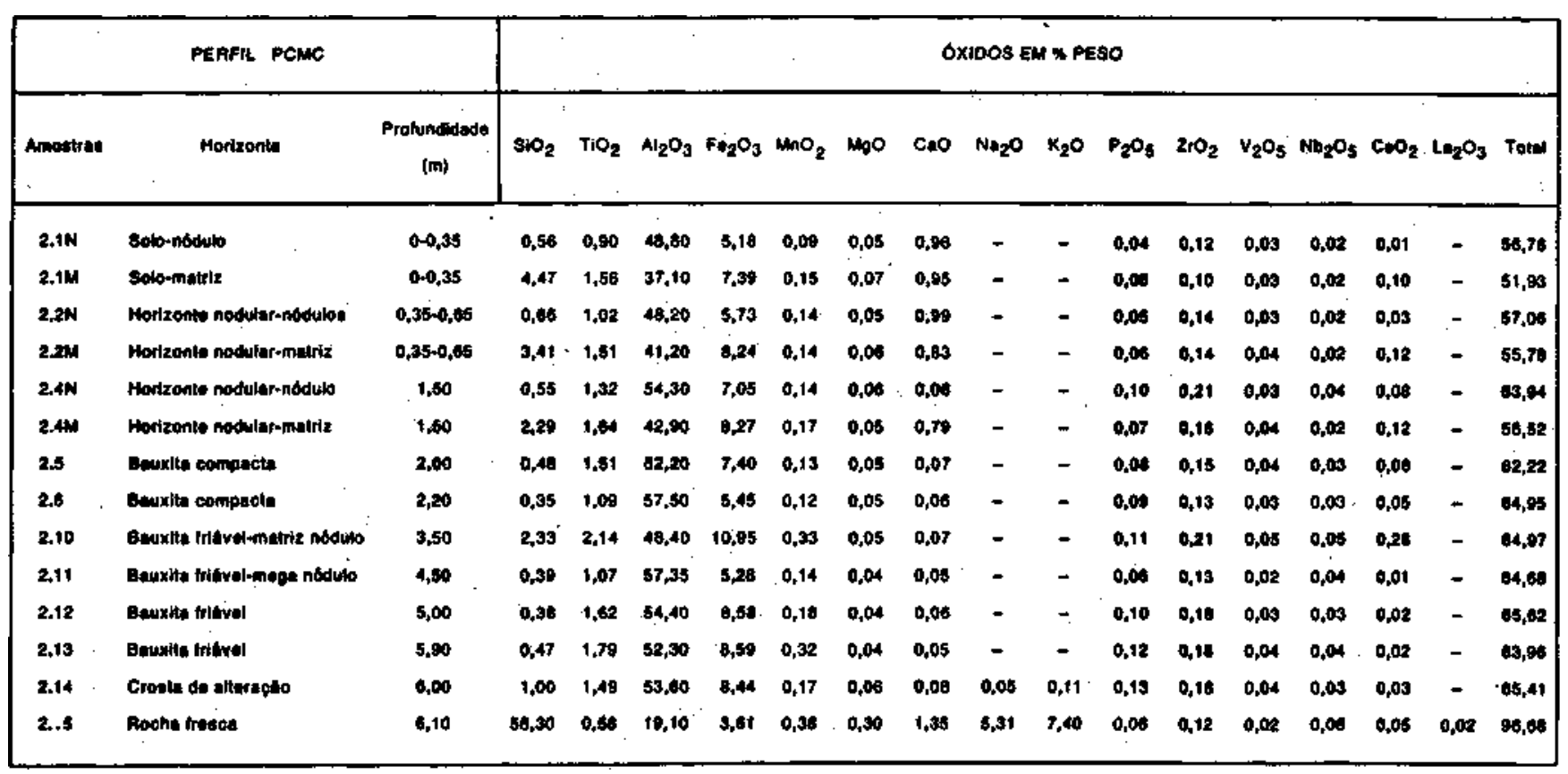

essencialmente, pela existência de duas grandes unidades de organização:

- dominantemente, existe um mosaico de grandes cristais de gibbsita (10 a $30 \mu \mathrm{m})$, que se embricam entre si. No meio desses cristais e dispersos nesse cristaliplasma gibbsítico, observam-se numerosos granules, que apresentam, ao microscópio petrográfico, as seguintes características: tamanho semelhante ao dos cristais de gibbsita; coloração vermelhoalaranjada; relevo forte; forma arredondada ou ligeiramente geométrica (hexagonal, retangular, com ângulos arredondados); isótropos sob luz polarizada ou, mais raramente birrefringentes, com coloração análoga àquela que possuem sob luz normal; e freqüentes zonação e fissuras;

- subordinadamente, ocorre um sistema de grandes poros (mais desenvolvido no horizonte friâvel e mais restrito no coerente), onde se podem observar dois tipos de cutanas: um, forma plasma análogo, nas suas propriedades, aos grânulos precedentes (plasma vermelho-alaranjado), que é geralmente isótropo em luz polarizada, mas que as vezes pode ser birrefringente; o outro, constitui plasma de coloração marrom-avermelhada, comumente zonado (sugerindo depósito iluvial), isótropo ou fracamente birrefringente e com relevo fraco.

O plasma vermelho-alaranjado pode ocorrer isolado (caso menos freqüente) e formar grandes volumes homogêneos, recortados por fissuras, tipo fendas de dissecação. O mais freqüente, entretanto, é encontrá-lo associado ao plasma marrom-avermelhado, de maneira não bem definida, mas com limites nítidos entre ambos. Em geral, nota-se, no plasma marrom-avermelhado, a existência de uma franja de pequenos cristais de gibbsita, que evidencia a forma do poro.

Comportamento do zircônio no perfil bauxítico As análises químicas apresentadas na tabela 4 mostram que

Tabela 5 - Balanço geoquímico isovolume. Porcentuais de perdas e ganhos dos elementos em relação à rocha original, durante as primeiras fases de alteração do tinguaito do Morro do Cristo. As composições químicas das amostras encontram-se na tabela 4

Table 5 - Isovolume balance. Losses and gains percentages of elements, related to the parent material during the first step of bauxitization of the Morro do Cristo tinguaite. Chemical analyses are shown in table 4

\begin{tabular}{|c|c|c|c|c|c|c|c|c|c|c|c|c|c|c|c|c|}
\hline \multirow{2}{*}{ Amostra } & \multirow{2}{*}{$\begin{array}{l}\text { Dengidado } \\
\text { apurento }\end{array}$} & \multicolumn{15}{|c|}{ 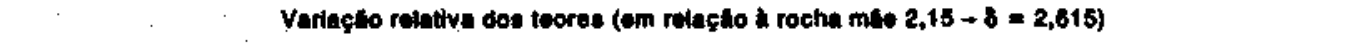 } \\
\hline & & $\mathbf{S i O}_{2}$ & $\mathrm{mo}_{2}$ & $\mathrm{Al}_{2} \mathrm{O}_{3}$ & $\mathrm{Fe}_{2} \mathrm{O}_{3}$ & $\mathrm{MnO}_{2}$ & $\mathrm{CaO}$ & Mgo & Nago & $\mathrm{K}_{2} \mathrm{O}$ & $\mathrm{v}_{2} \mathrm{O}_{5}$ & $\mathrm{Nb}_{2} \mathrm{O}_{5}$ & $\mathrm{P}_{2} \mathrm{O}_{5}$ & $\mathrm{C}_{0} \mathrm{O}_{2}$ & $\mathrm{~L}_{2} \mathrm{O}_{3}$ & $\mathrm{ZrO}_{2}$ \\
\hline 2,12 & 1,196 & $-89,7$ & 26,0 & 26,8 & 6,3 & $-78,8$ & $-90,1$ & $-09,9$ & $-100,0$ & $-100,0$ & $\mathbf{5 , 4}$ & $-8,3$ & $-32,2$ & $-75,7$ & $-90,3$ & $-34,4$ \\
\hline 2.13 & 1,055 & $-99,7$ & 25,1 & 10,5 & $-3,6$ & $-80,1$ & $-96,5$ & $-94,3$ & $-100,0$ & $-100,0$ & 0,5 & $-81,4$ & $-24,6$ & $-79,8$ & $-91,2$ & $-40,6$ \\
\hline 2.14 & 1,185 & $-90,2$ & 17,9 & 28,2 & 7,3 & $-79, a$ & $-97,3$ & $-01,1$ & $-99,6$ & $-90,3$ & 2,4 & $-81,8$ & $-3,7$ & $-0,4$ & $-80,1$ & $-31,1$ \\
\hline
\end{tabular}


o Zr permanece, em todos os horizontes, com teores aproximadamente constantes. Com o objetivo de verificar seu comportamento durante a alteração ferralítica, foi aplicado nas amostras do horizonte bauxita friável, que possuem a estrutura original da rocha preservada $(2.12,2.13$ e 2.14$)$, o raciocínio isovolume (Millot \& Bonifas 1955) (Tab. 5).

Os resultados da tabela 5 mostram que $\mathrm{Si}, \mathrm{Ca}, \mathrm{Mg}, \mathrm{Na}$ e $\mathrm{K}$ são quase completamente eliminados, desde o início da alteração. Mn, Nb, La e Ce exibem alta mobilidade, apresentando altas taxas de exportação (70 a 90\%). O Zr, assim como o $\mathrm{P}$, apresenta mobilidade intermediária, com taxas de exportação entre 20 e $40 \%$, Fe, V, Al e Ti são praticamente imóveis, concentrando-se no perfil.

No que diz respeito ao $\mathrm{Zr}$, os dados da tabela 5 são significativos pois, considerado geralmente como elemento residual, verifica-se que possui notável mobilidade neste meio superficial, pelo menos no Início da alteração. Este fato está, sem dúvida alguma, ligado a três aspectos principais: a. nestas rochas, o essencial do estoque do $\mathrm{Zr}$ não se encontra no zircão, mineral praticamente ausente nos tinguaítos, mas associado a zirconossilicatos cálcio-sódicos complexos, mais facilmente hidrolizáveis; b. existência de valores elevados de $\mathrm{pH}$ nas águas que percolam estas rochas alcalinas (valores acima de 9,0 já foram encontrados em certos lençóis freáticos do planalto de Poços de Caldas), facilitando a solubilização do Zr; c. riqueza em $\mathrm{F}$ do mineral zirconífero. $\mathrm{O}$ íon $\mathrm{F}^{-}$é um complexante importante para o $\mathrm{Zr}$ e outros elementos.

Uma boa parte do $\mathrm{Zr}$ contido no tinguaíto fica assim eliminado desde o início da hidrólise da rocha. Com respeito à expressão mineralógica do $\mathrm{Zr}$ no perfil bauxítico, os resultados obtidos na tabela 6 (coeficientes de correlação linear interelementos) evidenciam estreita associação do $\mathrm{Zr}$ com Fe, V, $\mathrm{Nb}, \mathrm{Ti}, \mathrm{Mn}$ e Ce. Isto, sem dúvida, indica, claramente, que o $\mathrm{Zr}$ deve encontrar-se associado, principalmente, à fase ferruginosa do minério de alumínio.

Estudos químicos pontuais, realizados por meio de microssonda, mostram que a gibbsita não é zirconífera, podendo entretanto exibir teores elevados em Fe e baixos em $\mathrm{Ti}$ e $\mathrm{Nb}$. A goethita, sempre associada a produtos mal identificados e impossível de ser isolada, parece apresentar teores elevados de $\mathrm{Ti}, \mathrm{Nb}$ e $\mathrm{Zr}$ em sua constituição, fato este constatado, também, em goethita da jazida de bauxita de Lages, Santa Catarina (F. Cesbron, comunicação oral). Entretanto, a maior quantidade do $\mathrm{Zr}$ nestas bauxitas parece ocorrer nos granules e nos dois tipos de plasma ferri-aluminoso, onde teores de até 2,5\% de $\mathrm{ZrO}_{2}$ podem ser detectados. Conforme os cálculos de correlação efetivados, notáveis quantidades de Ti, $\mathrm{Nb}$ e Ce acompanham o $\mathrm{Zr}$ nesses produtos.

Os dados apresentados na tabela 7 evidenciam que estes diferentes produtos amorfos ou com fraca organização (geles) são essencialmente de dois tipos: um, rico em $\mathrm{Al}$ e pobre em $\mathrm{Fe}, \mathrm{Ti}, \mathrm{Nb}$ e $\mathrm{Zr}$ (plasma marrom-avermelhado que ocorre associado ao sistema poral da bauxita); outro, ao contrário, rico em $\mathrm{Fe}, \mathrm{Ti}, \mathrm{Nb}$ e $\mathrm{Zr}$ (granules e plasma vermelho-alaranjado, que se encontram nos interstícios deixados pelos cristais de gibbsita e nos grandes poros). Estes geles zirconíferos, muito ricos em Fe, são fortemente hidratados (cerca de 40\% de $\mathrm{H}_{2} \mathrm{O}$ ).

CONSIDERACÕES FINAIS Os resultados químicos e mineralógicos obtidos do perfil de intemperismo do depósito de alumínio do Morro do Cristo evidenciam que os altos teores em $\mathrm{Zr}$ apresentados pelas bauxitas de Poços de Caldas representam apenas parte do estoque da rocha original, pois cerca de $40 \%$ do $\mathrm{Zr}$ foi lixiviado do perfil, nos primeiros estágios de evolução do processo de bauxitização. Sem dúvida, este comportamento, considerado até certo ponto anormal, só pode ser explicado pelo fato do $\mathrm{Zr}$ não se encontrar sob a forma de zircão na rocha original, mas sim fazendo parte da estrutura de um fluoro-zirconossilicato cálcio-sódico, ("giannettita"). Este mineral, facilmente alterável, libera o $\mathrm{Zr}$ que, em contato com um meio altamente alcalino, pode formar com o íon $\mathrm{F}^{-}$, também liberado pelo processo hidrolítico, complexo de grande estabilidade, que possibilita sua migração.

Por outro lado, os resultados mostram ainda a existência de uma íntima associação do $\mathrm{Zr}$ com a fase ferruginosa da bauxita. Além de parte do conteúdo total do $\mathrm{Zr}$ poder estar adsorvido ou mesmo fazer parte da estrutura da goethita, o essencial do estoque deste elemento na bauxita aparece incorporado a geles, altamente hidratados, de Fe, Al, Ti e Nb.

Tabela 6 - Coeficientes de correlação linear interelementos, calculados em 24 amostras das diferentes fácies da bauxita do Morro do Cristo

Table 6 - Interlements linear correlation coefficients, calculated from 24 samples of the various bauxitic facies

\begin{tabular}{|c|c|c|c|c|c|c|c|c|c|c|c|c|c|}
\hline & $k$ & 1 & 2 & 3 & 4 & 5 & 6 & 7 & s & 9 & 10 & 11 & 12 \\
\hline$J$ & & $\mathrm{ZrO}_{2}$ & $\mathrm{Fe}_{2} \mathrm{O}_{3}$ & $\mathrm{~A}_{2} \mathrm{O}_{3}$ & $\mathrm{sHo}_{2}$ & $\mathrm{CaO}$ & & $V_{2} O_{5}$ & $\mathrm{Hb}_{2} \mathrm{O}_{5}$ & $\mathrm{C}_{2} \mathrm{O}_{2}$ & $\mathrm{MnO}_{2}$ & $\pi O_{2}$ & $\mathrm{P}_{2} \mathrm{O}_{5}$ \\
\hline 1 & $2 \mathrm{rO}_{2}$ & & 0,017 & $0-, 048$ & $-0,084$ & $-0,436$ & $-0,136$ & 0,918 & 0,918 & 0,697 & 0,695 & 0,899 & 0,799 \\
\hline 2 & $\mathrm{~F}_{2} \mathrm{O}_{3}$ & & & $-0,320$ & 0,249 & $+0,274$ & 0,006 & 0,986 & 0,839 & 0,097 & 0,042 & 0,802 & 0,763 \\
\hline 3 & $\mathrm{Al}_{2} \mathrm{O}_{3}$ & & & & $-0,787$ & $-0,723$ & $-0,347$ & $-0,276$ & 0,119 & $-0,336$ & $-0,140$ & $-0,286$ & $0,+92$ \\
\hline 4 & $\mathrm{SHO}_{2}$ & & & & & 0,397 & 0,460 & 0,183 & $-0,121$ & 0,200 & 0,058 & 0,271 & $-0,014$ \\
\hline 5 & $\mathrm{CaO}$ & & & & & & 0,324 & $-0,303$ & $-0,564$ & $-0,172$ & $-0,335$ & $=0,361$ & $-0,721$ \\
\hline 6 & $M g O$ & & & & & & & $-0,036$ & $-0,188$ & $-0,008$ & $-0,122$ & $-0,081$ & $-0,004$ \\
\hline 7 & $\mathrm{~V}_{2} \mathrm{O}_{5}$ & & & & & & & & 0,876 & 0,954 & 0,921 & 0,977 & 0,765 \\
\hline 6 & $\mathrm{Nb}_{2} \mathrm{O}_{5}$ & & & & & & & & & 0,868 & 0,898 & 0,840 & 0,804 \\
\hline 9 & $\mathrm{CeO}_{2}$ & & 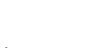 & & & & & & & & 0,697 & 0,913 & 0,628 \\
\hline 10 & $\mathrm{MnO}_{2}$ & & & & & & & & & & $\therefore$ & & 0,738 \\
\hline 11 & $\mathrm{TiO}_{2}$ & & & & & & & & & & & & 0,797 \\
\hline 12 & $\mathrm{P}_{2} \mathrm{O}_{5}$ & & & & & & & & & & & & \\
\hline
\end{tabular}


Tabela 7 - Composição química, em \% peso, dos produtos amorfos (geles) da bauxita do Morro do Cristo. Médias de várias análises realizadas com dois programas analíticos

Table 7 - Chemical composition, in weight percent, of the amorphous products found in the "Morro do Cristo" bauxite. Mean values of various analyses made with two analytical programs

\begin{tabular}{|c|c|c|c|c|c|c|c|c|c|c|c|c|c|c|c|c|}
\hline & $\mathrm{sio}_{2}$ & $\mathrm{TH}_{2}$ & $\mathrm{Al}_{2} \mathrm{O}_{3}$ & $\mathrm{~F}_{42} \mathrm{O}_{3}$ & Mno & MoO & $\mathrm{CuO}$ & $\mathrm{Na}{ }_{2} \mathrm{O}$ & $\mathrm{K}_{2} \mathrm{O}$ & $\mathrm{P}_{2} \mathrm{O}_{5}$ & $\mathrm{ZrO}_{2}$ & $\mathrm{Nb}_{2} \mathrm{O}_{5}$ & $\mathrm{C}_{2} \mathrm{O}_{3}$ & $\mathrm{Y}_{2} \mathrm{O}_{3}$ & $\mathbf{F}$ & cl \\
\hline Granuloe volmotho-distantado & 1,29 & 3,92 & 10,75 & 49,20 & 0,00 & 0,01 & 0,02 & 0,00 & 0,01 & 0,37 & 1,50 & 0,23 & 0,05 & 0,01 & 0,01 & 0,02 \\
\hline$n$ & 21 & 21 & 21 & 21 & $\theta$ & 8 & $\bullet$ & - & 6 & 13 & 21 & 21 & $\theta$ & • & 8 & e \\
\hline Plosme vermatho-elaranjudo & 1,87 & 5,35 & $11, \Delta 3$ & 39,39 & $0,0 \mathrm{~s}$ & 0,01 & 0,02 & 0,00 & 0,10 & 0,39 & 1.95 & 0,54 & 0,60 & 0,02 & 0,07 & 0,04 \\
\hline ต & 13 & 13 & 13 & 13 & 4 & 4 & 4 & 4 & 4 & 9 & 13 & 13 & 4 & 4 & 4 & 4 \\
\hline Planame marron-avermathado & 0,72 & 1,82 & $4,4,31$ & 12,42 & 0,01 & 0,01 & 0,02 & 0,01 & 0,04 & 0,13 & 0,73 & 0,20 & 0,35 & 0,01 & 0,09 & 0,02 \\
\hline$n$ & 9 & 9 & 9 & .9 & 3 & 3 & 3 & 3 & 3 & 6 & $\theta$ & 9 & 3 & 3 & 3 & 3 \\
\hline
\end{tabular}

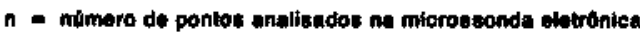

\section{REFERÊNCIAS BIBLIOGRÁFICAS}

AARDEN, H.M. \& GITTINS, J. 1974. Hiortdahlite from Kipawa river, Villedieu Township, Temiscaming country. Quebec, Canada. Can. Minerai, 12:241-247.

ADAMS, J.A.S. \& RICHARDSON, F.A, 1960. Thorium, uranium and zirconium concentrations in bauxite. Econ. Geol, 21:1653-1675

AMARAL, G.; BUSHEE, J.; CORDANI, U.G.; KAWASHITA, K.; REIHOLDS, J.H. 1967. Potassium-argon ages of alkaline rocks from southern Brazil. Geoch. Cosmoch. Acta, 31:117-142.

BROOKINS, D.G. 1988. Eh-pH diagrams for geochemistry. New York, Spring-Verlag. 176p

DEGENHARDT, H. 1957. Untersuchungen zur geochemischen verteirang der zirconium in der lithosphare. Geochim. Cosmochim. Acta 11:279.

ELLERT, R. 1959. Contribuição à geologia do maciço alcalino de Poços de Caldas. Bol Fac. Fil. Ciên. Letras USP, 237 (Geol. 18):5-63.

EMBREY, P.O. \& FULLER, J.P. 1980. A manual of new mineral names 1892 1978 Oxford, British Museum and Oxford University Press. 467p.

FREDERICKSON, A.F. 1948. Mode of occurence of titanium and zirconium in laterites. Am. Mineral 33:374

GOLDICH, S.S. 1938. A study in rock weathering. J. Geol., 46:17-58.

GOLDSCHMIDT, V.M. 1937. The principles of distribution of chemical elements in minerals and rocks. J. Chem. Soc., 1:655.

GOLDSCHMIDT, V.M. 1954. Geochemistry. In: MUIR, A. ed. Oxford, Clarendon Press.

GUIMARÃES, D. 1948. The zirconium ore deposits of the Poços de Caldas Plateau, Brazil and zirconium geochemistry. Belo Horizonte, Instituto de Tecnologia Industrial, p. 45-79. (Bol. 6).
LEVINSON, A.A. 1980. Introduction to exploration geochemistry. 2ed. USA, Applied Publ. Ltd. 924 p.

MILNES, A.R. \& FITZPATRICK, R.W. 1989. Titanium and zirconium minerals. 2 ed. In: DIXON, J.B. \& WEED, S.B. eds. Minerals in soil environment. Soil Sci. Soc. Amet. 1244p.

MERLINO, S. \& PERCHIAZI, N. 1988. Modular mineralogy in the cuspidine group of minerals. Can. Mineral. 26:933-943.

MILLOT, O. \& BONIFAS, M. 19SS. Transformations isovolumétriques dans les phénomenes de lateritisation et bauxitisation. Bull. da. Serv. de la Carte Geol d'Alsace et-de Lorraine, 8(1):3-22.

RONOV, A.B.; VA1NSHTEIN, E.E.; TUZOVA, A.M. 1961. Geochemistry of hafnium, zirconium and some other hydrolysate elements in clays. Geochemistry, 4:343-355.

STRUNZ, H. 1966. Mineralogische Tabellen. Leipzig, Akademiche Verlags Gesellschaft. 560p.

ZAKRUTKIN, V.E. \& SHVETSIVA, I.V. 1974. Zircon in the bauxites of the southern Timan region. Litologiya I Poleznye Iskopaemye, 5:32-40.

ZEISSINK, H.E. 1971. Trace element behaviour in two nickeliferous laterite profiles. Chem. Geol, 7:25.

Manuscrito A630

Recebido em 16 de novembro de 1989 Revisão do autor em 17 de janeiro de 1991 Revisão aceita em 4 de fevereiro de 1991 